\title{
Multiple Primary Invasive Small-Diameter Melanomas: Importance of Dermoscopy and Digital Follow-up
}

\author{
Gabriel Salerni ${ }^{1,2}$, Carlos Alonso ${ }^{1,2}$, Ramón Fernández-Bussy ${ }^{1}$
}

\begin{abstract}
1 Hospital Provincial del Centenario de Rosario, Argentina, \& Faculty of Medicine, Universidad Nacional de Rosario, Argentina 2 Diagnóstico Médico Oroño, Rosario, Argentina
\end{abstract}

Key words: melanoma, dermoscopy, skin cancer, imaging techniques

Citation: Salerni G, Alonso C, Fernández-Bussy R. Multiple primary invasive small-diameter melanomas: Importance of dermoscopy and digital follow-up. Dermatol Pract Concept. 2019;9(1):69-70. DOI: https://doi.org/10.5826/dpc.0901a16

Published: January 31, 2019

Copyright: @2019 Salerni et al. This is an open-access article distributed under the terms of the Creative Commons Attribution License, which permits unrestricted use, distribution, and reproduction in any medium, provided the original author and source are credited.

Funding: None.

Competing interests: The authors have no conflicts of interest to disclose.

Authorship: All authors have contributed significantly to this publication.

Corresponding author: Gabriel Salerni, MD, PhD, Bv. Oroño 1515, CP 2000, Rosario, Argentina. Email: gabrielsalerni@hotmail.com

\section{Introduction}

Early recognition is the most effective intervention to improve the prognosis of patients with melanoma. Dermoscopy is a noninvasive clinical examination technique that has been shown to increase both sensitivity and specificity in the clinical diagnosis of melanoma. Nevertheless, melanoma may be clinically but also dermoscopically indistinguishable from melanocytic nevi, making recognition a diagnostic challenge, especially in early stages. The comparison of sequential dermoscopic images of melanocytic lesions in search of subtle changes over time has been helpful in the diagnosis of early melanomas, which might lack specific criteria for malignancy [1]. In addition, the use of baseline regional photographs might facilitate the detection of new lesions, and visual changes in preexisting lesions, by providing a comparative reference for subsequent examinations [2].

\section{Case Presentation}

We present a case of a 47-year-old woman diagnosed with multiple primary invasive small-diameter melanomas. Melanoma 1 (Figure 1A), located on the right leg, was detected at the first visit in March 2015. It was a light-brown macule with a diameter of $3.1 \mathrm{~mm}$. Dermoscopy revealed atypical pigment network with irregular streaks and negative pigment network. Histology reported Breslow $0.2 \mathrm{~mm}$ thickness. Melanoma 2 (Figure 1B) was identified as a new lesion by body-mapping comparison at the first follow-up visit (Figure 2A) in October 2017. Melanoma 2 was $2.6 \mathrm{~mm}$ in diameter with atypical pigment network and grayish coloration on dermoscopy. Histology informed $0.4 \mathrm{~mm}$ Breslow thickness. Melanomas 3 and 4 (Figure 1, C and D), located on the back and the abdomen, respectively, were diagnosed in March 2018 after changes were observed during digital monitoring (Figure 2, B and C). Both lesions were less than $5 \mathrm{~mm}$ in diameter, and dermoscopy revealed typical reticular pattern in melanoma 3 and a quite regular globular pattern in melanoma 4. The decision to excise resulted only from the subtle dermoscopic changes observed. Histology informed Breslow thickness of 0.3 and $0.6 \mathrm{~mm}$, respectively.

\section{Conclusions}

Early recognition is the most effective intervention to improve melanoma prognosis. Several strategies such as the $\mathrm{ABCD}(\mathrm{E})$ 


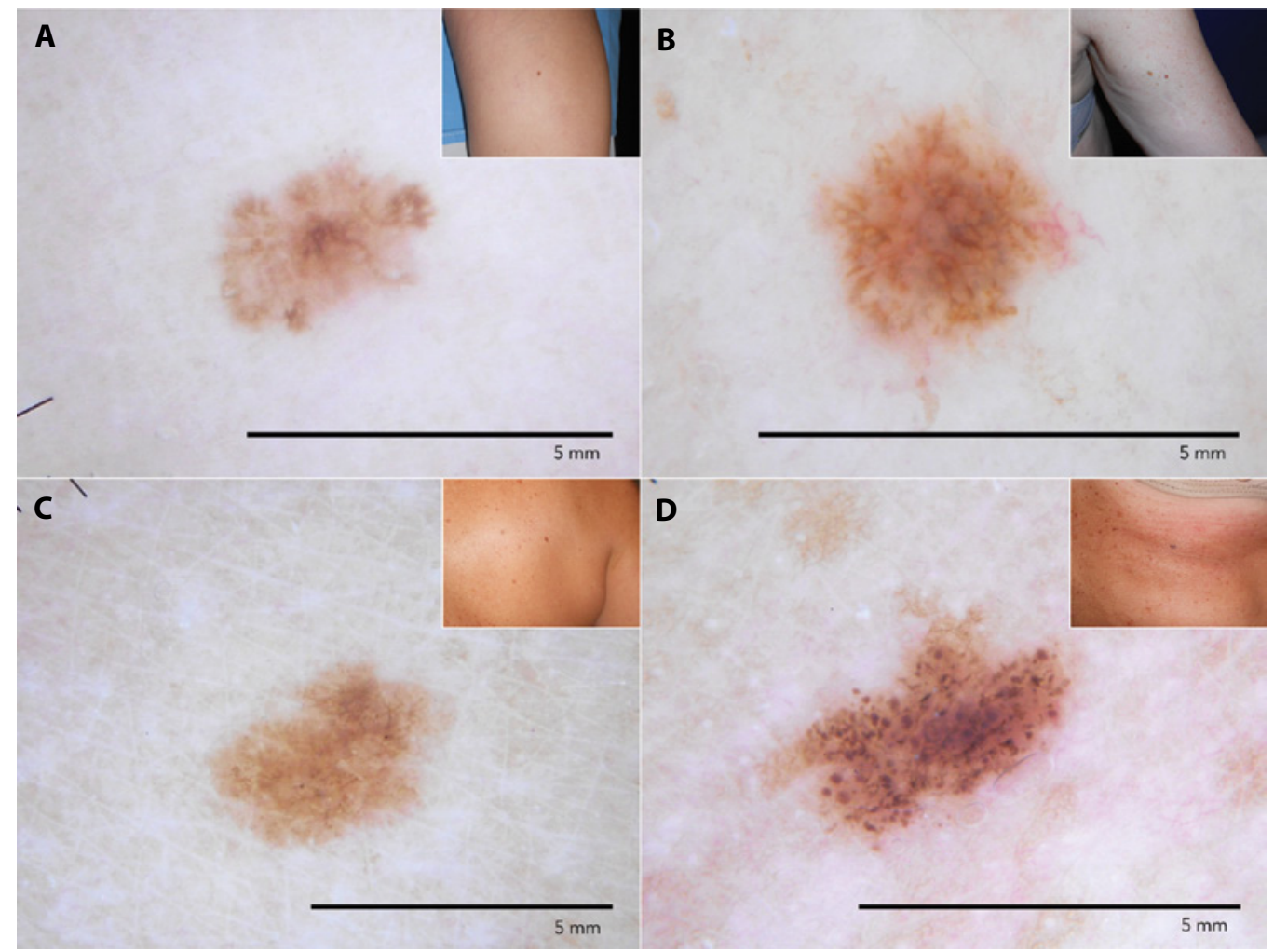

Figure 1. Clinical aspect of the 4 melanomas diagnosed, all with a diameter of less than $5 \mathrm{~mm}$. (A) Melanoma Breslow $0.2 \mathrm{~mm}$ located on the right leg, diagnosed at baseline. (B) Melanoma Breslow $0.4 \mathrm{~mm}$ located on the right arm detected during the first follow-up control. (C, D) Melanomas located on the back and abdomen, 0.3 and $0.6 \mathrm{~mm}$ Breslow thickness, respectively. [Copyright: (C2019 Salerni et al.]

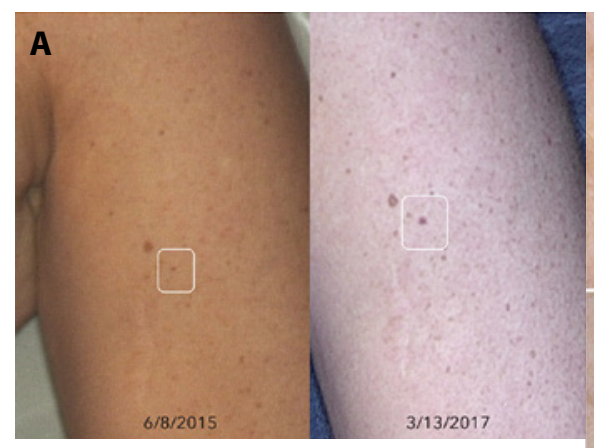

Figure 2. (A) Body-mapping comparison that allowed for the detection of melanoma 2 as a new lesion. (B, C) Dermoscopic changes lead-

ing to excision in melanomas 3 and 4. [Copyright: (C2019 Salerni et al.]

acronym, the "ugly duckling sign," or the EFG for the recognition of nodular melanoma ( $\mathrm{E}$ for elevation, $\mathrm{F}$ for firm, and $\mathrm{G}$ for growth), just to mention a few, have been proposed to enhance clinical recognition of atypical lesions that should undergo excision or close monitoring, but their usefulness in the detection of melanoma at early stages is questionable. The routine use of dermoscopy allows the detection of melanomas of which patients are unaware.

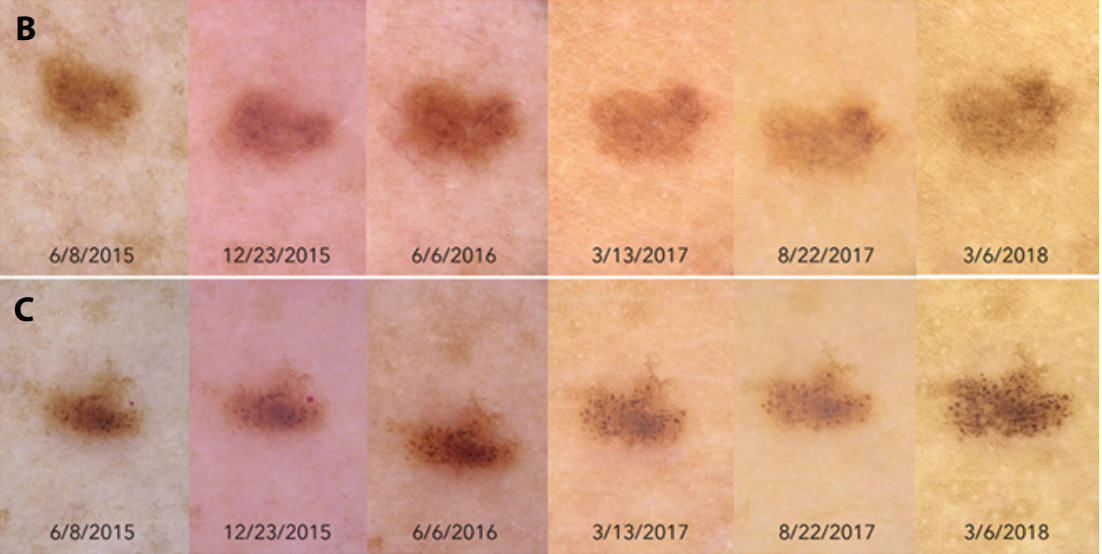

In the context of high-risk patients, the combined use of total-body photography and sequential digital dermoscopy enables the detection of incipient melanomas that might have been overlooked if assessed solely by the naked eye.

\section{References}

1. Salerni G, Carrera C, Lovatto L, et al. Benefits of total body photography and digital dermoscopy ("two-step method of digital follow-up") in the early diagnosis of melanoma in high-risk patients. $J \mathrm{Am}$ Acad Dermatol. 2012;67(1):e17-e27.

2. Salerni G, Terán T, Alonso C, FernándezBussy R. The role of dermoscopy and digital dermoscopy follow-up in the clinical diagnosis of melanoma: clinical and dermoscopic features of 99 consecutive primary melanomas. Dermatol Pract Concept. 2014;4(4):39-46. 